Cahiers de philosophie de l'université de

\title{
Critique d'art et pensée esthétique : questions de lignes
}

\section{Anne Elisabeth Sejten}

\section{OpenEdition}

1 Journals

Édition électronique

URL : https://journals.openedition.org/cpuc/633

DOI : $10.4000 /$ cpuc.633

ISSN : 2677-6529

Éditeur

Presses universitaires de Caen

\section{Édition imprimée}

Date de publication : 31 décembre 2014

Pagination : 71-86

ISBN : 978-2-84133-512-1

ISSN : $1282-6545$

\section{Référence électronique}

Anne Elisabeth Sejten, «Critique d'art et pensée esthétique : questions de lignes », Cahiers de philosophie de l'université de Caen [En ligne], 51 | 2014, mis en ligne le 13 juin 2018, consulté le 31 janvier 2023. URL : http://journals.openedition.org/cpuc/633; DOI : https://doi.org/10.4000/cpuc.633

\section{(c) $(1)(9$}

Creative Commons - Attribution - Pas d'Utilisation Commerciale 4.0 International - CC BY-NC 4.0 https://creativecommons.org/licenses/by-nc/4.0/ 


\title{
Critique d'art et pensée esthétique: questions de lignes
}

\begin{abstract}
J un Des attraits les Plus fascinants de l'esthétique de Diderot reside dans son aspect vital: le philosophe participe à sa construction,
avant même qu'on ne puisse parler d'esthétique. Innommée, mais active et édifiante, l'esthétique de Diderot se présente à nous comme une conceptualisation en chantier, parfois tâtonnante, mais tendue vers des perspectives d'élucidation. N'oublions pas que le terme d'esthétique vient à peine d'être inventé au milieu du siècle par le philosophe allemand Baumgarten qui, en 1750, publie son Aesthetica ${ }^{1}$, ouvrage qui marque l'inauguration de cette «science neuve». Et ce n'est qu'avec la troisième Critique de Kant, à la fin du siècle, donc après la mort de Diderot, que l'esthétique recevra une assise épistémologique bien circonscrite, place tout de même importante, puisqu'elle maintient et menace à la fois le projet criticiste en opérant ce fameux "libre jeu entre l'imagination et l'entendement $»^{2}$ que Kant associe au jugement du beau. Or, Diderot ne serait-il pas une présence philosophique naviguant, à sa manière originale, entre ces deux pôles de l'esthétique naissante de la seconde moitié du XVIII siècle? Célébrer le tricentenaire de Diderot, c'est aussi reconstruire et faire découvrir le chemin propre d'une pensée esthétique qui, lorsqu'elle est appréhendée aujourd'hui, ne manque pas de surprendre par son actualité. Il sera donc question ici de repérer quelques indices de la mise en mouvement de Diderot vers une philosophie qu'il convient de qualifier d'esthétique, tout en effleurant quelques échos postérieurs.
\end{abstract}

1. A. G. Baumgarten, Esthétique, J.-Y. Pranchère (trad. fr.), Paris, L'Herne, 1988.

2. E. Kant, Critique de la faculté de juger, A. Philonenko (trad. fr.), Paris, J. Vrin (Bibliothèque des textes philosophiques), 1984, p. 122. 


\section{L'intérêt esthétique en philosophie}

Lorsqu'on aborde l'œuvre monumentale de Diderot, il est effectivement difficile de ne pas être sensible aux multiples glissements par lesquels le philosophe prépare à l'esthétique, y aspire, comme si les problèmes philosophiques qui le préoccupent étaient intrinsèquement en attente d'une pensée esthétique. Cela est vrai dès les premiers écrits de jeunesse ${ }^{3}$. Déjà en 1746, dans Les Pensées philosophiques, Diderot déclare la supériorité de la quête de la vérité sur son acquisition. «On doit exiger de moi que je cherche la vérité, non que je la trouve ${ }^{4}$, déclare-t-il, dans l'un des passages les plus cités de ce premier ouvrage philosophique, et l'on peut, justement, se demander si Diderot n'a pas pressenti très tôt une attitude spécifiquement esthétique dans un tel usage «cherchant» de la raison, à savoir la capacité de procéder sans concepts, comme dira Kant 5 .

En 1749, la Lettre sur les aveugles renferme, bien au-delà du simple pamphlet religieux qui lui coûtera le séjour douloureux à Vincennes, une exploration enivrante du corps sensible. En passant par la perception des aveugles, Diderot insiste précisément sur un travail de la connaissance qui ne relève pas de l'entendement ou de l'esprit. Les aveugles inspirent à Diderot d'expérimenter non l'évidence du monde objectif et visible, mais un monde en train de se constituer par le toucher et le corps sentant. Telle sera sa réponse originale au "problème de Molyneux » : l'œil voit avant que l'esprit ne juge. Or, cette valorisation de la sensation comme source autonome de la connaissance ne représente qu'un premier pas vers l'esthétique.

Deux ans plus tard, la Lettre sur les sourds et muets poursuit l'idée d'une "anatomie métaphysique», inaugurée par les aveugles, s'organisant à nouveau au voisinage de questions nettement esthétiques. À leur tour, les sourds et muets fournissent à Diderot des arguments pour déconstruire tout un débat entre grammairiens et philosophes sur les inversions, voire sur l'ordre du discours, afin de poser en réalité le problème du caractère polyphonique et intraduisible dont relève l'expression artistique. C'est ainsi que Diderot va tracer des liens inédits entre l'expression de l'art et le sujet qui sent et qui pense, voire entre esthétique et épistémologie, en proposant notamment le concept de «hiéroglyphe poétique», qui relèvera précisément, dans l'ordre de la signification, d'une production de sens inséparable des matériaux et formes d'expression.

3. Nous renvoyons à notre précédent ouvrage: Diderot ou le Défi esthétique. Les écrits de jeunesse, 1746-1751, Paris, J. Vrin, 1999.

4. D. Diderot, Les Pensées philosophiques, «Pensée XXIX», in Euvres philosophiques, M. Delon (éd.), Paris, Gallimard (Bibliothèque de la Pléiade), 2010, p. 16.

5. «Est beau ce qui plaît universellement sans concept»; E. Kant, Critique..., p. 62. 
S’ajoute à ces ouvertures précoces vers une pensée esthétique, l'article «Beau» de l'Encyclopédie. Diderot le rédige juste après, sinon parallèlement, à la Lettre sur les sourds et muets, et dès le début, égal à lui-même, il insiste sur le caractère philosophique, voire «difficile» du beau, dont l'aspect familier est trompeur:

Avant que d'entrer dans la recherche difficile de l'origine du beau, je remarquerai d'abord, avec tous les auteurs qui en ont écrit, que par une sorte de fatalité, les choses dont on parle le plus parmi les hommes, sont assez ordinairement celles qu'on connaît le moins; et que telle est entre beaucoup d'autres, la nature du beau ${ }^{6}$.

Pourtant, dans l'article de l'Encyclopédie, Diderot ne semble avancer aucune thèse qui lui soit propre. Il passe en revue les sources antiques et contemporaines du beau, sources auxquelles il se dérobe. Bien sûr, on ressent l'intérêt qu'il peut prendre à la définition de la beauté comme "unité» chez saint Augustin, à la notion de «plaisir» chez Wolff, au «sentiment» né à l'occasion de l'œuvre d'art chez Crouzas, au critère du «sens interne» proposé par Hutcheson, à tous les «beaux doubles» du père André. Reste que c'est la thèse de la «perception des rapports » à laquelle il adhère sans vraiment l'élaborer. On ferait tout de même remarquer à quel point Diderot perçoit ce critère, qui était fortement dans l'air du temps (chez Rousseau et Rameau notamment ${ }^{7}$ ), sous un angle matériel et concret:

Quand je dis donc qu'un être est beau par les rapports qu'on y remarque, je ne parle point des rapports intellectuels ou fictifs que notre imagination $y$ transporte, mais des rapports réels qui y sont, et que notre entendement y remarque par le secours de nos sens ${ }^{8}$.

Il faudra cependant attendre le grand projet des Salons, l'émergence du critique d'art dans son face-à-face avec l'art, pour que la réflexion esthétique se munisse d'autres ressorts. C'est là la grande différence entre Diderot et Kant. Si la déduction transcendantale du jugement du beau se fait dans l'absence aveuglante des œuvres d'art, la réflexion esthétique de Diderot s'en nourrit et s'épanouit dans ce rapport particulier que le critique d'art établit forcément avec les œuvres qu'il commente. N'est-ce pas l'immense mérite de son ami Grimm de l'avoir poussé à poser un regard beaucoup plus attentif au Salon que celui de «la foule des oisifs »: "C’est là tâche que

6. D. Diderot, art. «Beau» de l'Encyclopédie, in CEuvres esthétiques, P. Vernière (éd.), Paris, Garnier, 1968, p. 391.

7. Voir l'article de Marian Hobson dans le présent ouvrage.

8. D. Diderot, art. «Beau» de l'Encyclopédie, in Euvres esthétiques, p. 425. 
vous m'avez proposée qui a fixé mes yeux sur la toile et qui m'a fait tourner autour du marbre ${ }^{9}$. Car c'est grâce à l'acuité de ce regard que Diderot s'est mis à réfléchir; d'où la gratitude qu'il exprime envers Grimm: «Si j'ai quelques notions réfléchies de la peinture et de la sculpture, c'est à vous, mon ami, que je les dois ${ }^{10}$.

C'est ainsi que les Salons se présentent à nous comme une œuvre à mille entrées, s'autorisant du seul regard attentif sur les œuvres, intégrant à bâtons rompus les idées circulant sur l'art et le beau à l'époque, le critique s'adressant au lecteur, aux peintres, et surtout à lui-même, les Salons s'approfondissant année après année. De ce tissu hétérogène, il en résulte plusieurs esthétiques, à plusieurs étages. L'une, Diderot ne la dit moins qu'il l'est; il la vit, il l'incarne, il la dresse en l'exemple qu'il donne. Alors que cette esthétique s'organise autour du sujet, se référant aux sensations et sentiments du critique d'art, une autre surgirait bien davantage au sein des considérations sur le travail artistique, sur le faire, sur ce que c'est que de faire un tableau. Cette esthétique, Diderot la signe en l'accueillant à part, en la raffinant en marge des Salons, que ce soit sous forme de commentaires dispersés au cours des Salons, ou dans les essais qu'il a pris soin d'attacher à quelques-uns de ses Salons. Relevons d'abord sommairement quelques indices du premier type d'esthétique pour ensuite nous arrêter de façon plus détaillée sur le second, celui qui est promu par le philosophe lui-même lorsqu'il se place du côté de la production artistique.

\section{Le sujet, critère esthétique assurant de l'universel}

C'est en décrivant les tableaux et les sculptures dont il se fait juge que le critique d'art se découvre penseur en esthétique. Passant allégrement, parfois sévèrement, d'un tableau à l'autre, Diderot retrouve sa liberté d'écrivain, sans avoir à penser aux conditions de systématicité et d'argumentation logique imposées par le travail collectif de l'Encyclopédie. Il est vrai que les circonstances l'encouragent à donner libre cours à sa verve d'écrivain, à peindre en quelque sorte à son tour les tableaux exposés, puisqu'il écrit pour les lecteurs distingués, peu nombreux, de la Correspondance littéraire, ces hauts personnages étrangers qui n'ont pas vu les tableaux eux-mêmes. Face au défi de décrire les œuvres d'art, de passer des tableaux au langage, des images aux mots, les stratégies de Diderot sont multiples. Tantôt il dramatise, tantôt il rêve, pour évoquer

\footnotetext{
9. D. Diderot, Salons, II. Salon de 1765, E. M. Bukdahl et A. Lorenceau (éd.), Paris, Hermann, 1984 , p. 21.

10. Ibid.
} 
la poésie des tableaux qu'il aime. Simuler des promenades à la place des tableaux de Vernet n'en est que l'exemple le plus connu et le plus étalé.

L'essentiel, c'est cependant que les nombreuses descriptions au moyen desquelles les Salons grandissent démesurément et s'imposent comme une œuvre littéraire, dispersée et énorme, mettent Diderot en contact avec lui-même comme celui qui juge du beau. Le temps passé au milieu des toiles et des marbres correspond à une sensibilisation du sujet qui en subit les effets: "J'ai donné le temps à l'impression d'arriver et d'entrer. J'ai ouvert mon âme aux effets, je m'en suis laissé pénétrer ${ }^{11}$. S'attarder aux œuvres pour que celles-ci parlent directement à l'âme, voilà enfin l'expérience dont se fortifie le critique d'art. Il doit passer par lui-même, par l'état d'âme dans lequel les œuvres d'art l'auront mis, pour ensuite faire revivre les tableaux pour lui et ses lecteurs. Dans ce sens, Diderot se fait le poète des Salons. Qu'un tableau ait été en mesure de déclencher la réécriture, au moyen de laquelle le critique d'art se l'approprie, est en soi un signe de sa valeur esthétique.

Il est frappant de voir que Charles Baudelaire élève au rang de critère critique cette traduction des tableaux par l'écriture poétique, lorsque, un siècle plus tard, il poursuit la tâche de critique d'art au Louvre. Dans son premier Salon, celui de 1846 , qui comprend en introduction le texte programmatique «À quoi bon la critique?», Baudelaire affirme que «la meilleure critique est celle qui est amusante et poétique», et que "le meilleur compte rendu pourra être un sonnet ou une élégie $»^{12}$. Abstraction faite de l'intérêt que Baudelaire accorde aux synesthésies, le critère esthétique qu'il retient, c'est le sentiment du sujet sensible et cultivé. Le tableau est digne de recevoir l'épithète de «beau» lorsqu'il est pour ainsi dire renvoyé, reflété, résonné par un autre mode d'expression artistique - par la poésie à laquelle il aura invité. Cette réécriture s'autorise du sentiment du sujet: «le beau exprimé par le sentiment», ou bien «réfléchi par un esprit intelligent et sensible» ${ }^{13}$. Les critères d'ordre subjectifs - Baudelaire réclame une critique "partiale» et «passionnée» - sont paradoxalement la condition pour que la critique d'art soit juste et bien fondée. À l'occasion du Salon de 1855, il le dit explicitement: «je me suis contenté de sentir $»^{14}$, position humble et naïve, sorte d'asile contre les esprits académiques et leurs hiérarchies de genre, mais qui garantit la "plus abondante impartialité» ${ }^{15}$.

11. Ibid.

12. C. Baudelaire, Salon de 1846, in Euvres complètes, C. Pichois (éd.), Paris, Gallimard (Bibliothèque de la Pléiade), t. II, 1976, p. 418.

13. Ibid.

14. C. Baudelaire, Exposition universelle (1855), in CEuvres complètes, t. II, p. 578.

15. Ibid. 
Bien qu'il y ait dans cette promotion du sentir et du sentiment comme critère, une réaction «romantique» contre un académisme trop rigoureux, récusé aussi par Diderot, il est tout de même révélateur que le sentiment soit élevé au rang d'argument esthétique. Lorsque Diderot traite tardivement "[d]e la critique» dans ses Pensées détachées sur la peinture, la sculpture, l'architecture et la poésie, il a également, de façon laconique et interrogative, mis la critique au compte du seul sentiment: «Je voudrais bien savoir où est l'école où l'on apprend à sentir ${ }^{16}$. Si le sentir est invoqué ici, c'est que l'œuvre d'art évoque autre chose que sa matérialité. Elle donne lieu à un sentiment - et c'est dans la particularité de ce sentiment que le beau esthétique se loge. Et même si Diderot, face à l'expérience esthétique, pas plus que Baudelaire après lui, n'en détermine les traits spécifiques, l'appel au sentir rappelle celui ultérieur et rigoureux de Kant. Dès le premier paragraphe de la troisième Critique, Kant affirme que si le jugement de goût ne peut pas être «logique» mais «esthétique», c'est précisément parce que son "principe déterminant ne peut être que subjectif» ${ }^{17}$.

Pourtant, et cette fois-ci en contraste avec Baudelaire, Diderot semble beaucoup plus avancé un siècle plus tôt, plus philosophique aussi, lorsqu'il s'agit de penser à partir de l'art plastique envisagé dans sa présence matérielle et concrète. D'un Salon à l'autre, il est initié aux questions techniques de la peinture et il s'occupe de plus en plus des techniques de l'art $^{18}$. Il commence à faire le lien entre les sentiments que les œuvres évoquent en lui et leur exécution. Toute une esthétique, dès lors, s'édifie du côté des œuvres, faisant appel à un vocabulaire de lignes, couleurs, composition, etc. Évoquons le cas, très commenté, de Chardin.

\section{Chardin, peintre, coloriste... et magicien}

Il est significatif que Chardin, dans le Salon de 1763, soit salué pour sa seule profession de peintre, comme si c'était là la clef de sa grandeur, le secret de

16. D. Diderot, Pensées détachées sur la peinture, la sculpture, l'architecture et la poésie; pour servir de suite aux "Salons", in Salons, IV. Héros et martyrs. Salons de 1769, 1771, 1775, 1781 - Pensées détachées sur la peinture, la sculpture, l'architecture et la poésie; pour servir de suite aux "Salons", E. M. Bukdahl et al. (éd.), Paris, Hermann, 1995, p. 385.

17. E. Kant, Critique..., $\$ 1$, p. 49 (nous soulignons).

18. On évoque souvent, en vue d'une classification des Salons, les Salons de 1761 et de 1763 comme les Salons d'apprentissage, les grands Salons de 1765 et de 1769 comme ceux de l'épanouissement et de la maturité, et enfin les derniers Salons comme témoignant du déclin et du désintérêt de Diderot. Voir aussi E. M. Bukdahl, Diderot, critique d'art, Copenhague, Rosenkilde et Bagger, t. I, Théorie et pratique dans les "Salons" de Diderot, J.-P. Faucher (trad. fr.), 1980. 
son art: «C'est celui-ci qui est un peintre, c'est celui-ci qui est un coloriste ${ }^{19}$. C'est que les natures mortes de Chardin font preuve de «magie ${ }^{20}$; elles recréent pour ainsi dire les choses: "C'est la nature même», s'écrie-t-il. «Les objets sont hors de la toile et d'une vérité à tromper les yeux» ${ }^{21}$. Pourtant, la production picturale demeure d'autant plus magique et énigmatique - «On n'entend rien à cette magie» - qu'elle relève des «couches épaisses appliquées les unes sur les autres ${ }^{22}$. Achèvement et dissolution représentatifs cohabitent dans les toiles de Chardin, d'où l'exercice auquel Diderot invite son lecteur: «Approchez-vous, tout se brouille, s'aplatit et disparaît. Éloignez-vous, tout se recrée et se reproduit ${ }^{23}$. Et une fois ce regard myope jeté sur la matérialité du tableau, le commentaire va en quelque sorte en deçà des couleurs, se rabattant au niveau de leurs matières premières:

Ô Chardin, ce n'est pas du blanc, du rouge, du noir que tu broies sur ta palette; c'est la substance même des objets, c'est l'air et la lumière que tu prends à la pointe de ton pinceau, et que tu attaches sur la toile ${ }^{24}$.

Au milieu des déesses et draperies qui peuplent la plupart des tableaux exposés au Louvre - surtout les plus prestigieux de la peinture religieuse, mythologique ou historique, dont Diderot sait apprécier le grand art -, l'auteur sait cependant tirer de Chardin une leçon qui se rapproche de la phénoménologie de la perception chez Maurice Merleau-Ponty deux siècles plus tard. Le fameux «doute de Cézanne» dont parle Merleau-Ponty, consiste précisément dans la double articulation, chez Cézanne, entre la chose qu'il fait voir et ce dont est faite la représentation, entre le visible et de l'invisible. Cézanne voulait, dit-il, «peindre la matière en train de se donner forme ${ }^{25}$. Or, Chardin, dans la mise en scène de Diderot, crée également du visible au moyen de tout ce qu'on ne voit pas: la lumière, les ombres, jusqu'à l'insignifiance de chaque touche, de chaque ligne appliquée à la toile. Cela rappelle le mot de Paul Klee cité par Merleau-Ponty dans L'CEil et l'Esprit: «la couleur [...] n'imite plus le visible, elle rend visible ${ }^{26}$. Or, n'est-ce pas aussi la naissance du visible au fond des matériaux que Diderot commence à entrevoir dans les porcelaines, cuivres et gibiers de Chardin?

19. D. Diderot, Salon de 1763, in Essais sur la peinture - Salons de 1759, 1761, 1763, G. May et J. Chouillet (éd.), Paris, Hermann, 1984, p. 219.

20. Ibid., p. 220.

21. Ibid., p. 219.

22. Ibid., p. 220.

23. Ibid.

24. Ibid.

25. M. Merleau-Ponty, «Le doute de Cézanne», in Sens et Non-sens, Paris, Nagel, 1966, p. 23.

26. M. Merleau-Ponty, L'Eil et l'Esprit, Paris, Gallimard, 1960, p. 74. 
Il est vrai que les transports enthousiastes à l'égard de Chardin occupent une position marginale dans le Salon de 1763, plus marginale encore dans les Salons pris dans leur ensemble. En revanche, les deux grands Salons de 1765 et de 1767 , nous semblent esquisser les éléments esthétiques qui nuancent l'isolement du cas de Chardin.

\section{Lignes multiples}

Rappelons que les Essais sur la peinture, joints au Salon de 1765 pour y « faire suite", ainsi que le préambule au Salon de 1767, marquent l'irruption, sinon des traités esthétiques, du moins des amorces d'une réflexion esthétique de la part de Diderot. Car à chaque fois que Diderot se met à écrire un «supplément», c'est signe de son engagement philosophique, ou plutôt d'un manque philosophique. Le Supplément au voyage de Bougainville démontre clairement cette dialectique chez Diderot, car si l'île de Tahiti avait été découverte géographiquement par le navigateur Bougainville, elle restait en attente de sa découverte philosophique. De la même façon, il s'agit peut-être, avec les suppléments à la critique d'art, de se défaire des théories insuffisantes que Diderot avait passées en revue dans son article «Beau», ainsi que de tirer des remarques détachées ici et là sous la plume du salonnier, des arguments plus solides et cohérents en vue d'une pensée esthétique. Les Essais sur la peinture représentent certainement le premier moment du véritable programme qu'il propose aux peintres. Car même si, à sa manière habituelle, il s'adresse au lecteur, il y va des questions pratiques du peintre: comment peindre, comment exécuter un tableau, d'après quel modèle et d'après quels préceptes? Et plus fondamentalement : comment voir en peinture?

Dans l'ouverture fulgurante, dialogique, des Essais sur la peinture, Diderot accable effectivement son lecteur d'une suite rapide de questions relatives aux portraits détaillés qu'il fait de deux pauvres personnages infirmes, la vieille femme aveugle et le bossu. Leur infirmité, que l'on croit à tort limitée aux organes concernés - aux yeux et au dos -, se laisse en réalité percevoir partout, dans la moindre partie de leur corps. Dans cette démonstration, Diderot fait solennellement appel à la nature, d'abord en ce qui concerne l'aveugle:

Mais appelez la nature, présentez-lui ce col, ces épaules, cette gorge, et la nature vous dira [...] que c'est une femme qui a perdu les yeux dans sa jeunesse ${ }^{27}$.

27. D. Diderot, Essais sur la peinture, in Essais sur la peinture - Salons de 1759, 1761, 1763, p. 11. 
Le portrait du bossu obéit à la même logique:

Couvrez cette figure, n'en montrez que les pieds à la nature; et la nature dira sans hésiter: Ces pieds sont ceux d'un bossu ${ }^{28}$.

La Nature, fortement allégorisée, est donc invoquée comme juge infaillible. Ces deux corps sont marqués de toutes parts par son œuvre et par le travail, lent, continu et presque imperceptible que la difformité leur a infligé. Son propre mode de production est assimilé à une sorte de vision. L'argumentation de Diderot est ici comparable à celle de la Lettre sur les aveugles, où les aveugles sont convoqués pour apprendre à ceux qui voient ce qu'est la vue. De même, la vieille femme aveugle et le bossu servent à dresser la Nature à la fois comme critère et maître pour ceux qui font de la peinture. Les peintres doivent apprendre à voir, comme l'aurait fait la nature. Le troisième exemple est symétrique des deux premiers, puisqu'il s'agit là d'une sculpture grecque. Placerait-on un nez tors sur cette sculpture sublime, ce nez serait démenti par toutes les autres parties du corps, parties qui auraient préparé et commandé le nez le plus délicieux de tous.

L'essentiel, c'est cependant que les descriptions des infirmes, comme celle de la beauté grecque, permettent à Diderot d'insister sur le critère de la nature tout en le modifiant. La nature est comme saisie de l'intérieur, au niveau des rapports organiques. Dès lors, imiter la nature signifie plutôt imiter la nature comme regard, comme procédé, comme moyen de procéder en art. En art, les lignes doivent obéir à un même principe de cohérence interne, à l'image du tissu organique dont est composée la nature. Aussi Diderot parle-t-il d'une "observation continue des phénomènes", pour qu'on prenne acte de la «liaison secrète» ou bien de «l'enchaînement nécessaire ${ }^{29}$. En tout cas, il doit y avoir une solidarité des lignes, les unes empiétant sur les autres pour que le tableau se tienne tout seul: «Tout est lié, tout tient ${ }^{30}$. Diderot exige une unité inébranlable: "Faites que je ne puisse ni arrêter mes yeux, ni les arracher de dessus votre toile ${ }^{31}$.

La métaphore du lien parcourt donc les nombreux conseils que Diderot adresse volontiers aux jeunes peintres. Aussi le «soyez observateurs " ${ }^{32}$ ne vise-t-il aucun réalisme, ce mot signifiant plutôt l'importance pour le peintre d'adopter une méthode analogue à la façon dont travaille la nature - méthode qu'il ne peut trouver autrement qu'en exerçant son art. Car si le peintre

\footnotetext{
28. Ibid.

29. Ibid., p. 21.

30. Ibid., p. 30.

31. Ibid., p. 43.

32. Ibid., p. 16.
} 
doit observer la nature afin d'apprendre à voir qu'elle n'est jamais égale à elle-même, mais se trouve en perpétuels mouvements et changements, il en va de même pour le dessin, qui doit être exercé comme ce qui produit des altérations. Par ce rapprochement, le dessin doit être appris hors de l'école et de ses "pauvres règles» 33 et "attitudes académiques qui déplaisent à la mort » ${ }^{34}$. Tout se passe comme s'il fallait aller en deçà de la représentation picturale pour expérimenter la véritable conspiration des lignes, voire «la conspiration générale des mouvements, conspiration qui se sent, qui se voit, qui s'étend et serpente de la tête aux pieds ${ }^{35}$.

On constate donc à quel point on est passé de la disposition des figures de la composition classique, à ce que Diderot nomme «un système de difformités bien liées et bien nécessaires ${ }^{36}{ }^{6}$, dont la vieille femme aveugle et l'homme bossu n'étaient que les premiers exemples pédagogiques. La figure, précisément, est conçue comme le déplacement d'une ligne : «le poco più ou poco meno, le trait en dedans ou en dehors fait défaut ou beauté ${ }^{37}$. Du même coup, l'imitation de la belle nature se voit reformulée au niveau des lignes qui sont en quelque sorte partout et qui ne sont nulle part. Si «l'homme de la beauté », selon Diderot, se trouve entre «l'enfance» et «la vieillesse ${ }^{38}$, c'est parce que les lignes de l'art le font surgir sur fond des lignes multiples de la vie.

Dès lors, on mesure mieux la portée esthétique de ces exemples, en vue d'élucider ce que nous nous proposons d'appeler ici une esthétique des lignes multiples. La vieille femme aveugle et le bossu sont tous deux considérés, bien que ce soit à la lumière d'une faute, d'une insuffisance corporelle, comme des systèmes organiques que chaque ligne soutient et reflète, participant toutes aux répercussions de la faute. Et une fois que ces lignes complices, solidaires et organiquement imbriquées les unes dans les autres, s'emparent du modèle de l'artiste, la nature pour ainsi dire change de nature, et elle change de place. Elle n'est plus figée en face du peintre, elle prend place dans son tableau à faire. La nature dynamisée agit désormais sous les mains du peintre. C'est d'ailleurs pourquoi le peintre est surpris, même parfois dépassé, par l'attendu qui se produit lorsqu'il croyait à tort appliquer l'idée qu'il avait en tête: «L'artiste qui prend la couleur sur sa palette, ne sait pas toujours ce qu'elle produira sur son tableau ${ }^{39}$.

\footnotetext{
33. D. Diderot, Essais sur la peinture, p. 12.

34. Ibid., p. 15.

35. Ibid.

36. Ibid., p. 75.

37. Ibid., p. 13.

38. Ibid.

39. Ibid., p. 20.
} 
Si Diderot, en fait, privilégie un vocabulaire technique, c'est que l'œuvre se cherche au niveau des effets matériels de son exécution:

En passant de la palette sur la scène entière de la composition, la couleur est modifiée, affaiblie, rehaussée et change totalement d'effet. Alors l'artiste tâtonne, manie, remanie, tourmente sa couleur ${ }^{40}$.

Une telle idée de la production artistique, rapprochée de la nature, conduit à penser une sorte de défiguration des figures. Les peintres doivent, à leur tour, opérer une défiguration du réel qui seule peut les conduire à briser les lignes et à trouver, au sein de leur organicité, ce que la nature ne montre nulle part. Car, au fond, Diderot rêve constamment de ces variations du peintre, altérant les lignes de sorte qu'elles aboutissent à une sorte d'idéalité et de vérité en peinture, et nous voici face au thème qui organisera le préambule au Salon de 1767, celui de la ligne idéale.

\section{Ligne idéale}

Deux ans plus tard, Diderot semble en effet avoir oublié toute référence aux lignes multiples. Une seule l'intéresse et le fait perdre haleine: «la ligne vraie; modèle idéal de la beauté ${ }^{41}$. Ce qui marque de façon massive le préambule au Salon de 1767, c'est de toute évidence l'éloge néoplatonicien du modèle idéal. Pourtant, là aussi, Diderot commence, en vue de penser ce qu'est cette ligne idéale, par déconstruire le credo de l'imitation de la belle nature en entamant le procès contre

ces gens qui parlent sans cesse de l'imitation de la belle nature, croient de bonne foi qu'il y a une belle nature subsistante, qu'elle est, qu'on la voit quand on veut, et qu'il n'y a qu'à la copier. Si vous leur disiez que c'est un être tout idéal, ils ouvriraient de grands yeux, ou ils vous riraient au nez ${ }^{42}$.

Cependant, Diderot ne va pas aussi loin que Platon, pour qui les œuvres d'art ne sont que l'ombre ou le reflet bien terne des Idées, le monde des Idées étant constitutivement non sensible. Force lui est bien au contraire d'insister sur le caractère très particulier de cette «nature» que les artistes doivent imiter. "Car quel est, au fond, le modèle d'après lequel tu peins? », ne cesse-t-il de demander au peintre. Se produit alors un espace dialogique, comme c'est souvent le cas, lorsque le philosophe demande son dû au

\footnotetext{
40. Ibid.

41. D. Diderot, Salon de 1767, in Salons, III. Ruines et paysages. Salon de 1767, E. M. Bukdahl et al. (éd.), Paris, Hermann, 1995, p. 70.

42. Ibid., p. 62.
} 
critique d'art au bout de tant d'heures passées avec des œuvres d'art et les peintres: le dialogue spectaculaire qui fait le nœud du préambule et qui va permettre à Diderot d'exposer l'ignorance éclatante du peintre, piégé par des arguments sortant d'un platonisme réinventé au point d'être méconnaissable. Car même si le peintre avoue que même la plus belle femme n'est pas la beauté, et qu'il prendrait sans doute les plus beaux bras d'une femme, les plus belles fesses d'une autre, etc., et que oui, à y penser, cet assemblage sélectif se ferait au nom du modèle légué par les œuvres des Anciens, il ne sait quoi répondre lorsque Diderot lui demande comment il se comporterait s'il n'y avait pas eu d'Antiquité. Le peintre se tait, et Diderot prend la parole à sa place pour essayer d'expliquer comment les Grecs, qui, eux, n'ont pas eu d'antiques eux-mêmes, ont pu trouver le modèle de la beauté. Du coup, le dialogue bascule dans le monologue du philosophe: «Écoute-moi donc, car je vais tâcher de t'expliquer comment les Anciens, qui n'avaient pas d'antiques, s'y sont pris $[\ldots] »^{43}$.

D'une manière assez typique de sa démarche, Diderot soumet la question du modèle antique à un morcellement temporel, ce qui lui permettra de penser par la suite, analogiquement, plusieurs moments différents du processus de travail artistique. Avant de devenir les Anciens, les Grecs ont cherché leurs moyens en art à travers une «longue observation» de la nature. Leur "premier pas» a consisté plus précisément dans des études de la détérioration de la nature, de son perpétuel déclin: «ils ont commencé par sentir les grandes altérations, les difformités les plus grossières, les grandes souffrances ${ }^{44}$. Les difformités des Essais sur la peinture réapparaissent donc dans ce retour imaginé aux Grecs au commencement de leur quête de la ligne idéale, ligne vraie, modèle de la beauté. Loin de souscrire au dogme académique des grands maîtres grecs ou de se retrancher dans un monde platonicien des Idées, Diderot retourne en réalité aux lignes multiples des Essais sur la peinture. Car même si la nature ne montre nulle part le modèle de la beauté, «ni total, ni partiel » - Diderot est formel là-dessus -, il faudra passer par elle et sa façon de produire. Or, la nature ne produit que des « ouvrages viciés » ${ }^{45}$, c'est-à-dire des ouvrages imperfectibles, usés par le temps.

Les lignes vivantes, mouvantes, s'alternant à l'infini, n'étaient donc pas si loin que ça. Au fond, c'est grâce à elles, aux lignes multiples, que Diderot parvient à repenser l'idée du modèle antique. Toujours est-il que sa petite leçon d'histoire imaginée des Grecs retombe sur des analogies

\footnotetext{
43. D. Diderot, Salon de 1767, p. 68.

44. Ibid., p. 69.

45. Ibid.
} 
relevant du processus de travail artistique. Les merveilles grecques ne se sont pas faites d'un seul coup; elles se doivent à un temps très long que Diderot décrit comme «une marche lente et pusillanime» et «un long et pénible tâtonnement ${ }^{46}$, pour suggérer ainsi le travail plein d'incertitudes et de difficultés que chaque artiste doit accomplir en exerçant son art. Expérimenter les lignes multiples, celles imbriquées les unes dans les autres, chacune vibrant avec toutes, apprendre à les voir, apprendre à les recréer en peinture, c'est le premier pas vers la ligne idéale. Car c'est à cette condition du premier pas qu'il est possible de passer, «avec le temps ${ }^{47}$, des lignes pour ainsi dire «empiriques » à la ligne idéale de l'art. Une sorte de renversement - «éloignement», dit Diderot - s'accomplit, lorsque l'artiste, muni de l'autorisation de la nature, est prêt à travailler contre la dégradation et la décomposition perpétuelles auxquelles sont soumises les lignes de la nature. C'est ainsi que Diderot peut dire des Grecs, tout en repassant par Platon, qu'ils «[...] s'éloignent sans cesse du portrait, de la ligne fausse, pour s'élever au vrai modèle de la beauté, à la ligne vraie» ${ }^{4}$.

Apprendre des Grecs, dans cette perspective, revient donc à trouver les critères de l'art, le modèle de la beauté, sa mesure et son échelle, au sein du processus de travail. La ligne idéale du Salon de 1767 surgit sur fond des lignes multiples des Essais sur la peinture. Les difformités ne faisaient que conduire au modèle de la beauté, cet être construit que Diderot décrit précisément dans le préambule en ces termes: « un homme ou une femme [qui serait] supérieurement propre à toutes les fonctions de la vie, et [qui serait] parvenu à l'âge du plus entier développement, sans en avoir exercé aucune ${ }^{49}$. En perpétuel mouvement, en perpétuel déclin aussi, les lignes de la nature feraient expérimenter, voire figurer, lorsqu'elles passent du côté des artistes, la ligne idéale autour de laquelle elles vibrent toutes.

\section{Lignes à suivre}

Observer la nature pour apprendre à voir et à dessiner les lignes qu'elle ne montre nulle part, mais qu'elle montre tout de même comme dans des éclairs divins, cette idée de faire croiser les lignes avec la ligne idéale, de tirer l'idéalité de la matérialité la plus complexe, la plus liée qu'il y ait, serait peut-être une des premières tentatives, dans la pensée esthétique moderne, pour aborder la question de la forme en art. Comme disait Paul Klee, «l'art

\footnotetext{
46. Ibid.

47. Ibid.

48. Ibid., p. 70.

49. Ibid., p. 68.
} 
consiste dans des questions de forme ${ }^{50}$. Une préoccupation majeure parcourant l'ensemble de l'esthétique moderne consiste certainement à penser forme et fond comme une relation inséparable dans l'œuvre d'art. Et, depuis Diderot, bien des philosophes et théoriciens de l'art se sont confrontés aux problèmes des lignes multiples. Contentons-nous d'en évoquer, en guise de conclusion, quelques affinités.

Il est intéressant de voir dans l'exemple majeur que représente Paul Valéry, un souci des lignes très semblable à la pensée de Diderot. En effet, dans son essai sur Degas daté de 1936, Valéry, lui aussi, se préoccupe de lignes. Il y raconte comment Ingres, idole du jeune Degas, avait commandé à celui-ci de dessiner des lignes: «Faites des lignes... Beaucoup de lignes, soit d'après le souvenir, soit d'après nature ${ }^{51}$. Car ce sont les lignes qui mènent à la forme qui, elle, mène aux idées: «la forme est féconde en idées ${ }^{52}$. L'essentiel, c'est précisément ce primat de la forme née des lignes agissant depuis l'intérieur du dessin, d'où cet autre mot assez analogue de Mallarmé, que Valéry cite dans le même essai: «ce n'est point avec des idées que l'on fait des vers... C'est avec des mots " ${ }^{53}$. Que ce soient les lignes du peintre ou les matériaux du langage ou d'un autre support sémiotique, l'artiste, qu'il soit poète ou peintre, doit se laisser guider par cette matière, ces unités de construction, et en tirer une œuvre d'art. Surtout, il ne faut se faire aucune illusion sur la possibilité de vouloir une œuvre d'art sans passer par les modes matériels de sa production.

Nous avons déjà esquissé, avec l'exemple de Chardin, l'affinité de Diderot avec une certaine phénoménologie de la perception. Or, Maurice Merleau-Ponty s'efforcerait sans doute d'entrevoir dans les lignes multiples l'apparition énigmatique de l'être. Dans cette perspective «ontologisante", aussi bien Chardin que Cézanne montreraient la constitution du monde, «la vibration des apparences, qui est le berceau des choses ${ }^{54}$. Dans la filiation de Merleau-Ponty, mais aussi en rupture avec lui, un philosophe comme Jean-François Lyotard serait sans doute plus attaché à une "éthique» des lignes. Car, comme il le dit à l'occasion des dessins et tableaux d'Adami: "La ligne porte une responsabilité "55. Les lignes caractéristiques d'Adami, qu'on voit presque trop facilement, sont solidaires de celles qui ont été

50. Paul Klee, Théorie de l'art moderne, P.-H. Gonthier (éd. et trad. fr.), Paris, Gallimard, 1998, p. 9.

51. P. Valéry, Degas Dessin Danse, in Euvres, J. Hytier (éd.), Paris, Gallimard (Bibliothèque de la Pléiade), t. II, 1960, p. 1187.

52. Ibid., p. 1207.

53. Ibid., p. 1208.

54. M. Merleau-Ponty, «Le doute de Cézanne», p. 30.

55. J.-F. Lyotard, Que peindre?, Paris, La Différence, 1987, p. 37. 
sacrifiées. Ainsi émane-t-il de la ligne qu'on voit ici et maintenant une voix à peine audible, "écoutant une foule de lignes possibles " ${ }^{56}$, celles effacées et qui n'ont jamais été portées jusqu'au point où elles peuvent faire corps, image, rythme, etc. D'où cette étrange capacité de la ligne à faire sentir l'imprésentable: "C'est pourquoi [la ligne] rappelle le néant, éveille le désert ${ }^{57}$.

Diderot, bien sûr, n'allait pas aussi loin, mais en relisant ses deux textes qui représentent des sommets réflexifs de l'aventure salonnière, on se rend compte à quel point il ne prépare pas seulement le terrain de la pensée esthétique qui est encore la nôtre, il en assume les premières étapes. Par leur dissymétrie remarquable - le plaidoyer pour les lignes multiples ici, l'éloge de la ligne idéale là -, les Essais sur la peinture et le préambule au Salon de 1767 font à la fois se confronter et s'unir, au plus près des œuvres sur le plan technique, deux esthétiques: l'esthétique des lignes multiples et l'esthétique de la ligne idéale. Mais Diderot savait aussi se laisser émouvoir par les œuvres d'art, et sonder les sensations et sentiments qu'elles évoquent en lui. De façon majestueuse, sa critique d'art oscille entre la matérialité picturale et plastique et les entités abstraites et idéales que sont la vérité et les idées. Parce que les deux aptitudes cohabitent dans sa critique, la spontanéité de l'amateur d'art se heurte à la sagacité du connaisseur. Sujet et objet doivent incessamment se corriger l'un l'autre, si la réflexion esthétique ne veut pas s'éluder dans un préromantisme, ni se figer dans le formalisme des concepts. C'est grâce à ses intuitions à la fois concrètes et métaphysiques, empiriques et idéales que Diderot se fait penseur de la nature énigmatique de l'art. Il est parmi les premiers à voir que le secret de l'œuvre d'art consiste dans le caractère inimitable de l'image - ou de l'expression artistique tout court, quel que soit son support. Diderot inaugure en réalité le procès contre le beau naturel en rapportant la ligne idéale aux lignes multiples.

Anne Elisabeth Sejten

Université de Roskilde

56. Ibid.

57. Ibid., p. 47. 